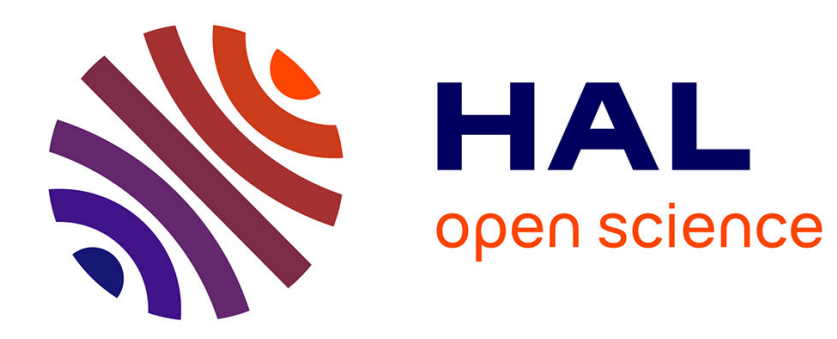

\title{
Doubly resonant multiphoton ionization
}

M. Crance

\section{- To cite this version:}

M. Crance. Doubly resonant multiphoton ionization. Journal de Physique Lettres, 1978, 39 (6), pp.67-73. 10.1051/jphyslet:0197800390606700 . jpa-00231445

\section{HAL Id: jpa-00231445 https://hal.science/jpa-00231445}

Submitted on 1 Jan 1978

HAL is a multi-disciplinary open access archive for the deposit and dissemination of scientific research documents, whether they are published or not. The documents may come from teaching and research institutions in France or abroad, or from public or private research centers.
L'archive ouverte pluridisciplinaire HAL, est destinée au dépôt et à la diffusion de documents scientifiques de niveau recherche, publiés ou non, émanant des établissements d'enseignement et de recherche français ou étrangers, des laboratoires publics ou privés. 


\title{
LE JOURNAL DE PHYSIQUE-LETTRES
}

\author{
Classification \\ Physics Abstracts \\ 32.00
}

\section{DOUBLY RESONANT MULTIPHOTON IONIZATION}

\author{
M. CRANCE
}

Laboratoire Aimé Cotton, C.N.R.S. II, Bât. 505, 91405 Orsay, France

(Reçu le 28 juin 1977, révisé le 27 décembre 1977, accepté le 16 janvier 1978)

\begin{abstract}
Résumé. - Nous étudions théoriquement un cas particulier d'ionisation multiphotonique doublement résonnante. Plus précisément, nous considérons deux niveaux quasi résonnants avec deux harmoniques successives de la fréquence du champ d'excitation. La méthode utilisée est fondée sur le formalisme des opérateurs effectifs introduit initialement par Armstrong, Beers et Feneuille dans l'étude de ce problème. Le résultat le plus intéressant est de montrer la possibilité d'observer des effets d'interférence importants sur les largeurs des courbes de résonance. Ce traitement nous est l'occasion de préciser la relation entre le formalisme des opérateurs effectifs et la théorie des perturbations.
\end{abstract}

\begin{abstract}
We investigate theoretically a particular case of doubly resonant multiphoton ionization. More precisely, we consider two levels quasi-resonant with two successive harmonics of the field frequency. The method used is based on the effective operator formalism first introduced for this problem by Armstrong, Beers and Feneuille. The main result is to show the possibility of observing large interference effects on the width of the resonances. Moreover this treatment allows us to make more precise the connection between effective operator formalism and standard perturbation theory.
\end{abstract}

1. Introduction. - In multiphoton ionization experiments, resonances are observed [1] when some atomic levels are quasi-resonant with some harmonics of the field frequency. Two types of theoretical treatments have been applied to this problem. The first approach is based on the use of standard perturbation theory with either a quantum [2] or a classical [1] description of the field. The second starts from the model initially introduced by Armstrong, Beers and Feneuille [3], who describe resonances in term of effective operators.

Multiple resonances can occur, in particular when some levels are approximately resonant with the same harmonic of the electromagnetic field frequency. In the latter case, one observes the interference between the various resonant paths consisting in a real absorption of $p$ photons, followed by a $(n-p)$ photon photoionization. Multiple resonances appear also in an other situation which occurs in noble gases for example. One level is resonant with the $p$ harmonic of the field frequency and an other one is resonant with the $(p+1)$-harmonic. When these two levels are connected by a strong electric dipole interaction, it is no longer possible to consider that two independent quantum paths interfere.

An approach of this problem was recently given by Faisal [4]. This author describes multiphoton ionization by a single quantum path process. Therefore, his treatment can not describe all the atomic situations.
We reconsider this problem by taking into account non resonant processes. The system is described by using the dressed atom picture. The basic equations giving the ionization probability are derived in section 2 . In section 3 we discuss the result obtained for continuous excitation when the mixing between the ground state and the quasi-resonant state is weak. This approximation would certainly be valid for optical multiphoton ionization of noble gases. This discussion leads to make more precise the structure of the effective operators introduced in the description of phenomena. This restatement of the question is given in section 4.

2. Basic equations. - We described multiphoton ionization in the vicinity of two successive resonances in the following way (see Fig. 1) :

- a non-resonant $n$-photon process induced by an effective interaction $H_{n}^{E}$ which can be obtained from perturbation theory to the $n$th order;

- a sequence of resonant processes involving :

- a $p$-photon transition from the ground state $\mathrm{i}$ to the quasi-resonant state $\varphi_{1}$ (induced by an effective interaction $H_{p}$ );

- a $(p+1)$-photon transition from the ground state to the quasi-resonant state $\varphi_{2}$ (induced by an effective interaction $H_{p+1}$ ); 


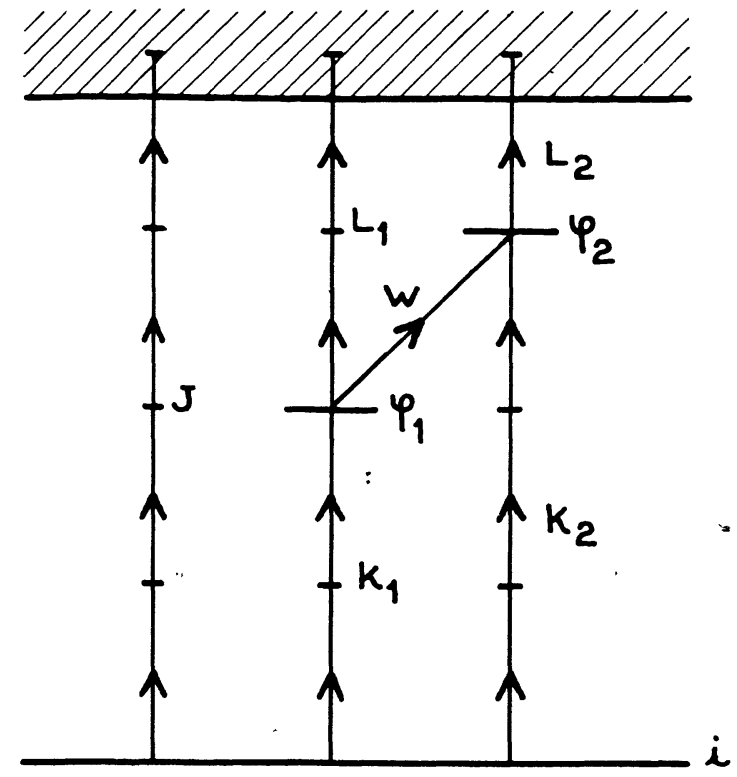

FIG. 1. - Schematic energy diagram.
- a $(n-p)$-photon transition from $\varphi_{1}$ to a continuum state $\Psi_{E}$ (induced by an effective interaction $\left.H_{n-p}^{E}\right)$;

- a $(n-p-1)$-photon transition from $\varphi_{2}$ to a continuum state $\Psi_{E}$ (induced by an effective interaction $H_{n-p-1}^{E}$ );

- a one-photon interaction $V$ between $\varphi_{1}$ and $\varphi_{2}$.

The system is described as a dressed atom. The Hamiltonian can be written

$$
H=H_{\mathrm{DA}}+H_{\mathrm{AF}}
$$

where $\mathcal{H}_{\mathrm{DA}}$ is a reduced Hamiltonian of the dressed atom and $\mathcal{H}_{\mathrm{AF}}$ is an effective interaction between the atom and the field. We consider only the unperturbed dressed atom states $|\mathrm{i} N\rangle,\left|\varphi_{1} N-p\right\rangle$, $\left|\varphi_{2} N-p-1\right\rangle,\left|\Psi_{E} N-n\right\rangle$ corresponding respectively to the states where the atom is in state $|\mathrm{i}\rangle$ (resp. $\left|\varphi_{1}\right\rangle\left|\varphi_{2}\right\rangle\left|\Psi_{E}\right\rangle$ ) and the field is in the state where $N$ photons (resp. $N-p, N-p-1, N-n$ ) are present in the $\omega$-mode, the other modes being empty

$\mathcal{H}_{\mathrm{DA}}$ can be written

$$
\begin{aligned}
\mathscr{H}_{\mathrm{DA}}=\hbar\left(\Omega_{\mathrm{i}}+N \omega\right)|\mathrm{i} N\rangle\langle\mathrm{i} N|+\hbar\left(\Omega_{1}\right. & +(N-p) \omega)\left|\varphi_{1} N-p\right\rangle\left\langle\varphi_{1} N-p\right|+ \\
& +\hbar\left(\Omega_{2}+(N-p-1) \omega\right)\left|\varphi_{2} N-p-1\right\rangle\left\langle\varphi_{2} N-p-1\right| \\
& +\int_{E_{\mathrm{I}}}^{\infty}(E+(N-n) \hbar \omega)\left|\Psi_{E} N-n\right\rangle\left\langle\Psi_{E} N-n\right| \mathrm{d} E
\end{aligned}
$$

where $\hbar \Omega_{\mathrm{i}}, \hbar \Omega_{1}, \hbar \Omega_{2}$ are the energies of the states $\mathrm{i}, \varphi_{1}, \varphi_{2}$ possibly light shifted by non resonant processes, $E_{\mathrm{I}}$ is the ionization energy of the atom. Under the usual rotating-wave approximation, in the space generated by $|\mathrm{i} N\rangle,\left|\varphi_{1} N-p\right\rangle,\left|\varphi_{2} N-p-1\right\rangle$ and $\left|\Psi_{E} N-n\right\rangle$ the only non vanishing matrix elements or $\mathcal{H}_{\mathrm{AF}}$ are

$$
\begin{aligned}
\left\langle\Psi_{E} N-n\left|H_{n}^{E}\right| \mathrm{i} N\right\rangle & =\left\langle\mathrm{i} N\left|H_{n}^{E}\right| \Psi_{E} N-n\right\rangle^{*}=\hbar J=\hbar j(N)^{n / 2} \\
\left\langle\Psi_{E} N-n\left|H_{n-p}^{E}\right| \varphi_{1} N-p\right\rangle & =\left\langle\varphi_{1} N-p\left|H_{n-p}^{E}\right| \Psi_{E} N-n\right\rangle^{*}=\hbar L_{1}=\hbar l_{1}(N)^{(n-p) / 2} \\
\left\langle\Psi_{E} N-n\left|H_{n-p-1}^{E}\right| \varphi_{2} N-p-1\right\rangle & =\left\langle\varphi_{2} N-p-1\left|H_{n-p-1}^{E}\right| \Psi_{E} N-n\right\rangle^{*}=\hbar L_{2}=\hbar l_{2}(N)^{(n-p-1) / 2} \\
\left\langle\varphi_{1} N-p\left|H_{p}\right| \mathrm{i} N\right\rangle & =\left\langle\mathrm{i} N\left|H_{p}\right| \varphi_{1} N-p\right\rangle^{*}=\hbar K_{1}=\hbar k_{1}(N)^{p / 2} \\
\left\langle\varphi_{2} N-p-1\left|H_{p+1}\right| \mathrm{i} N\right\rangle & =\left\langle\mathrm{i} N\left|H_{p+1}\right| \varphi_{2} N-p-1\right\rangle^{*}=\hbar K_{2}=\hbar k_{2}(N)^{(p+1) / 2} \\
\left\langle\varphi_{1} N-p|V| \varphi_{2} N-p-1\right\rangle & =\left\langle\varphi_{2} N-p-1|V| \varphi_{1} N-p\right\rangle=\hbar W=\hbar w(N)^{1 / 2}
\end{aligned}
$$

$j, k_{1}, k_{2}, l_{1}, l_{2}, w$ are mainly atomic parameters with a slow dependence on $\omega$. The $\omega$-dependence comes essentially from the energy denominators, appearing in the effective operators obtained by perturbation theory. Their corresponding variations remain negligible when the range of interest is very small compared to the frequency distance between the studied range of frequency and the nearest other resonances. We assume here that this condition is satisfied and we will neglect the frequency dependence of the effective operators. In the case of a single resonance, this assumption leads to a description of the ionization probability by a Fano profile. Such a profile is essentially characterized by an asymmetric resonance peak and a zero minimum. When the resonance is very strong, the minimum is very far from the resonant frequency and the assumption of frequency independent effective operators may be not valid in the vicinity of the minimum. In this sense, the profile obtained for a strong resonance may not be described by a complete Fano profile. This fact has been recently pointed out [5]. Nevertheless our assumption seems reasonable if the range of application is carefully limited. Numerical calculations have been carried out 
on 4-photon ionization of CsI near 6f resonance [6], in order to interpret experimental data [7]. The good agreement between experimental and calculated reso- nance profiles allows one to think that the use of frequency independent effective operators may be valid in some physical situations.

If we write the wave function under the following form :

$$
\begin{aligned}
& \cdot|\Psi(t)\rangle=\left\{a_{\mathrm{i}}|\mathrm{i} N\rangle+a_{1}\left|\varphi_{1} N-p\right\rangle+a_{2}\left|\varphi_{2} N-p-1\right\rangle+a_{E}\left|\Psi_{E} N-n\right\rangle\right\} \times \\
& \times \exp \left\{-i\left[\left(N-p-\frac{1}{2}\right) \omega+\frac{\Omega_{1}+\Omega_{2}}{2}\right] t\right\} .
\end{aligned}
$$

The equations of motion for the wave function components become

$$
\begin{aligned}
\mathrm{i} \dot{a}_{\mathrm{i}} & =\left(\left(p+\frac{1}{2}\right) \delta+\frac{\Delta}{2}\right) a_{\mathrm{i}}+K_{1}^{*} a_{1}+K_{2}^{*} a_{2}+\int J^{*} a_{E} \mathrm{~d} E \\
\mathrm{i} \dot{a}_{1} & =\left(\frac{\delta}{2}+\left(p+\frac{1}{2}\right) \Delta\right) a_{1}+K_{1} a_{\mathrm{i}}+W a_{2}+\int L_{1}^{*} a_{E} \mathrm{~d} E \\
\mathrm{i} \dot{a}_{2} & =-\left(\frac{\delta}{2}+\left(p+\frac{1}{2}\right) \Delta\right) a_{2}+K_{2} a_{\mathrm{i}}+W^{*} a_{1}+\int L_{2}^{*} a_{E} \mathrm{~d} E \\
\mathrm{i} \dot{a}_{E} & =-\delta_{E} a_{E}+J a_{\mathrm{i}}+L_{1} a_{1}+L_{2} a_{2}
\end{aligned}
$$

with

$$
\begin{aligned}
\Delta & =\frac{1}{2}\left\{\frac{\Omega_{1}-\Omega_{\mathrm{i}}}{p}-\frac{\Omega_{2}-\Omega_{\mathrm{i}}}{p+1}\right\} \\
\Omega & =\frac{1}{2}\left\{\frac{\Omega_{1}-\Omega_{\mathrm{i}}}{p}+\frac{\Omega_{2}-\Omega_{\mathrm{i}}}{p+1}\right\} \\
\delta & =\omega-\Omega \\
\delta_{E} & =\left(n-p-\frac{1}{2}\right) \omega-\frac{E}{\hbar}+\frac{\Omega_{1}+\Omega_{2}}{2} .
\end{aligned}
$$

The number of ions obtained is

$$
\mathcal{N}=P \int\left|a_{E}\right|^{2} \mathrm{~d} E
$$

where $P$ is the number of atoms interacting with the field. The system (4) is solved by first integrating over energy and then by solving differential equations. A general solution can be calculated for a square pulse excitation, without any assumption on the relative values of $J, K_{1}, K_{2}, L_{1}, L_{2}, W$. The formula is so complicated that it is quite unusable. We have preferred to solve eq. (4) in a realistic approximation and we study only continuous excitation. The assumption of resonant levels coupled more strongly with the continuum than with the ground level is actually valid in noble gases.

3. Solution in the case $W, L_{1}, L_{2} \gg K_{1}, K_{2} \gg J$. Since the transition $\varphi_{1}-\varphi_{2}$ is a one-photon process, it is reasonable to suppose that the interaction between these two levels is large. We study the case where $n-p \ll p$ and thus $L_{1}, L_{2} \gg K_{1}, K_{2} \gg J$.

Eq. (4) are solved in the approximation

$$
W, L_{1}, L_{2} \gg K_{1}, K_{2} \gg J .
$$

The number of ions obtained by unit-time is

$$
\mathcal{N}=2 P \frac{\left[J\left(p(p+1)\left(\tilde{\delta}^{2}-\tilde{\Delta}^{2}\right)-\tilde{W}^{2}\right)+\tilde{W}\left(\tilde{K}_{1} L_{2}+\tilde{K}_{2} L_{1}\right)+\tilde{K}_{1} L_{1} p(\tilde{\delta}-\tilde{\Delta})+\tilde{K}_{2} L_{2}(p+1)(\tilde{\delta}+\tilde{\Delta})\right]^{2}}{\left[p(p+1)\left(\tilde{\delta}^{2}-\tilde{\Delta}^{2}\right)-\tilde{W}^{2}\right]^{2}+\left[p(\tilde{\delta}-\tilde{\Delta}) L_{2}^{2}+(p+1)(\tilde{\delta}+\tilde{\Delta}) L_{1}^{2}+2 \tilde{W} L_{1} L_{2}\right]^{2}}
$$

$$
\begin{aligned}
\tilde{W} & =W+\frac{1}{\pi} \mathfrak{T} \int \frac{L_{1} L_{2}}{\hbar\left(\Omega_{\mathrm{i}}+n \omega\right)-E^{\prime}} \mathrm{d} E^{\prime} \\
\tilde{K}_{1} & =K_{1}+\frac{1}{\pi} \mathfrak{T} \int \frac{L_{1} J}{\hbar\left(\Omega_{\mathrm{i}}+n \omega\right)-E^{\prime}} \mathrm{d} E^{\prime}
\end{aligned}
$$

$$
\begin{aligned}
\tilde{K}_{2} & =K_{2}+\frac{1}{\pi} \iint \frac{L_{2} J}{\hbar\left(\Omega_{\mathrm{i}}+n \omega\right)-E^{\prime}} \mathrm{d} E^{\prime} \\
\tilde{\delta} & =\delta-\frac{1}{2}\left(\frac{\Delta^{L_{1}}}{p}+\frac{\Delta^{L_{2}}}{p+1}\right)+\frac{p+\frac{1}{2}}{p(p+1)} \Delta^{J}
\end{aligned}
$$




$$
\begin{aligned}
\tilde{\Delta} & =\Delta-\frac{1}{2}\left(\frac{\Delta^{L_{2}}}{p+1}-\frac{\Delta^{L_{1}}}{p}\right)-\frac{\Delta^{J}}{2 p(p+1)} \\
\Delta^{L_{1}} & =\frac{1}{\pi} \int \frac{L_{1}^{2}}{\hbar\left(\Omega_{\mathrm{i}}+n \omega\right)-E^{\prime}} \mathrm{d} E^{\prime} \\
\Delta^{L_{2}} & =\frac{1}{\pi} \mathfrak{T} \int \frac{L_{2}^{2}}{\hbar\left(\Omega_{\mathrm{i}}+n \omega\right)-E^{\prime}} \mathrm{d} E^{\prime} \\
\Delta^{J} & =\frac{1}{\pi} \mathfrak{T} \int \frac{J^{2}}{\hbar\left(\Omega_{\mathrm{i}}+n \omega\right)-E^{\prime}} \mathrm{d} E^{\prime} .
\end{aligned}
$$

Eq. (6) can be rewritten in a more elegant form by introducing a matrix $M$ defined by

$$
\tilde{M}^{-1}=\left(\begin{array}{cc}
p(\tilde{\delta}-\tilde{\Delta}) & -\tilde{\omega} \\
-\tilde{\omega} & (p+1)(\tilde{\delta}+\tilde{\Delta})
\end{array}\right)
$$

$$
\mathcal{N}=2 P \frac{\left[J+\left(\tilde{K}_{1} \tilde{K}_{2}\right) \tilde{M}\left(\begin{array}{l}
L_{1} \\
L_{2}
\end{array}\right)\right]^{2}}{1+\left[\left(L_{1} L_{2}\right) \tilde{M}\left(\begin{array}{l}
L_{1} \\
L_{2}
\end{array}\right)\right]^{2}} .
$$

For sake of simplicity the symbol $\sim$ will be omitted and all the transition matrix elements will be assumed to be real in this section.

The terms containing $J$ correspond to the direct ionization and to the interference between the direct process and the resonant ones. If we suppose that $J \ll K_{1}, K_{2}$ and neglect the terms containing. $J$, we keep only the terms corresponding to resonant processes, that is to say

$$
\mathcal{N}=2 P \frac{\left[(p+1) K_{1} L_{1}(\delta+\Delta)+p K_{2} L_{2}(\delta-\Delta)+W\left(K_{1} L_{2}+K_{2} L_{1}\right)\right]^{2}}{\left[p(p+1)\left(\delta^{2}-\Delta^{2}\right)-W^{2}\right]^{2}+\left[p(\delta-\Delta) L_{2}^{2}+(p+1)(\delta+\Delta) L_{1}^{2}+2 W L_{1} L_{2}\right]^{2}}
$$

We discuss this formula according to the relative values of the parameters $\Delta, L_{1}, L_{2}, W$. We first discuss the case of a well isolated resonance, which appears when the distance between the two resonances is larger than their expected widths $\left(|\Delta| \gg W_{1}, L_{1}, L_{2}\right)$. Secondly we discuss the case of weakly coupled quasiresonant level, and find again the situation already described, of two independent resonant quantum paths, each of them interfering with the non resonant quantum paths $\left(W \ll L_{1}, L_{2},|\Delta|\right)$. Finally we discuss the case of strongly coupled resonant levels. It allows us to stress the specific features due to the interferences between the two resonant quantum paths.

$3.1|\Delta| \gg W, L_{1}, L_{2}$ : WELL-ISOLATED RESONANCE. - From eq. (7), we can find again the result obtained for a well-isolated resonance. The resonance corresponding to $\varphi_{1}$ is obtained when $\delta=\Delta$, this resonance is well-isolated if $\delta+\Delta$ is very large along this resonant curve $\left(|\Delta| \gg W, L_{1}, L_{2}\right)$; then $\mathcal{N}$ becomes

$$
\mathcal{P}=2 P \frac{\left[K_{1}+\frac{W K_{2}}{(p+1)(\delta+\Delta)}\right]^{2}\left[L_{1}+\frac{W L_{2}}{(p+1)(\delta+\Delta)}\right]^{2}}{\left[p(\delta-\Delta)-\frac{W^{2}-L_{1}^{2} L_{2}^{2}}{(p+1)(\delta+\Delta)}\right]^{2}+\left[L_{1}+\frac{W L_{2}}{(p+1)(\delta+\Delta)}\right]^{4}}=\frac{2 P(K L)^{2}}{\delta_{0}^{2}+L^{4}}
$$

Eq. (8) is equivalent to the result obtained in [3] for a well isolated resonance. By going back to the content of each term of this equation, one can easily show by using standard perturbation theory this equivalence, term by term. Additional terms appear corresponding to high order processes which have to be neglected for consistency with the assumption that only the lowest non vanishing order of perturbation is taken into account.

We can already state explicitely the condition to obtain a well isolated resonance : $|\Delta| \gg W, L_{1}, L_{2}$. Conversely we can see that interference processes can be expected if this condition is not satisfied.

$3.2 W \ll L_{1}, L_{2},|\Delta| .-$ In this case $\mathcal{N}$ becomes

$$
2 P \frac{\left[(p+1) K_{1} L_{1}(\delta+\Delta)+p K_{2} L_{2}(\delta-\Delta)\right]^{2}}{p^{2}(p+1)^{2}\left(\delta^{2}-\Delta^{2}\right)^{2}+\left(p(\delta-\Delta) L_{2}^{2}+(p+1)(\delta+\Delta) L_{1}^{2}\right)^{2}} .
$$


Then, the resonant process consists of two independent channels. We should obtain a similar expression by considering two resonant levels $\varphi_{1}$ and $\varphi_{2}$ corresponding to harmonics $p$ and $q$, for any value of $p$ and $q$ satisfying the condition $p \gg n-p$ and $q \gg n-q$. The dependence of the resonant curves on the values of $K$ and $L$ is the same as in the case where $p=q$.

In fact we could have derived the equations describing doubly resonant photoionization for any harmonics $p$ and $q$ of the field; in that case we would have obtained :

$$
\begin{aligned}
\mathrm{i} \dot{a}_{\mathrm{i}} & =\left(\frac{p+q}{2} \delta+\frac{q-p}{2} \Delta\right) a_{\mathrm{i}}+K_{1}^{*} a_{1}+K_{2}^{*} a_{2}+\int J^{*} a_{E} \mathrm{~d} E \\
\mathrm{i} \dot{a}_{1} & =\left(\frac{q-p}{2} \delta+\frac{p+q}{2} \Delta\right) a_{1}+K_{1} a_{\mathrm{i}}+W a_{2}+\int L_{1}^{*} a_{E} \mathrm{~d} E \\
\mathrm{i} \dot{a}_{2} & =-\left(\frac{q-p}{2} \delta+\frac{p+q}{2} \Delta\right) a_{2}+K_{2} a_{\mathrm{i}}+W^{*} a_{1}+\int L_{2}^{*} a_{E} \mathrm{~d} E \\
\mathrm{i} \dot{a}_{E} & =-\delta_{E} a_{E}+J a_{\mathrm{i}}+L_{1} a_{1}+L_{2} a_{2}
\end{aligned}
$$

with

$$
\begin{aligned}
& \Delta=\frac{1}{2}\left\{\frac{\Omega_{1}-\Omega_{\mathrm{i}}}{p}-\frac{\Omega_{2}-\Omega_{\mathrm{i}}}{q}\right\} \\
& \Omega=\frac{1}{2}\left\{\frac{\Omega_{1}-\Omega_{\mathrm{i}}}{p}+\frac{\Omega_{2}-\Omega_{\mathrm{i}}}{q}\right\} \\
& \delta=\omega-\Omega \\
& \delta_{E}=\left(n-\frac{p+q}{2}\right) \omega-\frac{E}{\hbar}+\frac{\Omega_{1}+\Omega_{2}}{2}
\end{aligned}
$$

where $W$ is now an effective interaction if $|p-q|$ is larger than one.

The similarity of eq. (4) and $(9 b)$ can be understood by remembering that in terms of dressed atom multiply resonant photoionisation is described as a mixing of quasi degenerate levels. We can expect a large value of $W$ only when $|p-q|=1$ if $\varphi_{1}$ and $\varphi_{2}$ are connected by a dipolar electric interaction. In other case $W$ is either zero $(p=q)$ or is negligible $(|p-q|>1)$.
$3.3 W,|\Delta| \gg L_{1}, L_{2}$. - Since $W \gg L_{1}, L_{2}$, we can consider only the interaction $W$ and neglect $J$, $K, L_{1}, L_{2}$ in a first order calculation. Then the eigenstates of the system are now : $|\mathrm{i} N\rangle|E, N-n\rangle$ and

$$
\begin{aligned}
& |+\rangle=\cos \theta\left|\varphi_{1} N-p\right\rangle+\sin \theta\left|\varphi_{2} N-p-1\right\rangle \\
& |-\rangle=\sin \theta\left|\varphi_{1} N-p\right\rangle-\cos \theta\left|\varphi_{2} N-p-1\right\rangle
\end{aligned}
$$

with

$$
\begin{gathered}
\cos 2 \theta=\frac{\frac{\delta}{2}+\left(p+\frac{1}{2}\right) \Delta}{R} \\
\sin 2 \theta=\frac{W}{R} \\
R=\sqrt{W^{2}+\left(\frac{\delta}{2}+\left(p+\frac{1}{2}\right) \Delta\right)^{2}} .
\end{gathered}
$$

By using this eigenstate basis, $\mathcal{N}$ can be derived in a straight-forward way and we obtain :

$$
\mathcal{N}=2 P \frac{\left[K_{+} L_{+}\left[\left(p+\frac{1}{2}\right) \delta+\frac{\Delta}{2}+R\right]+K_{-} L_{-}\left[\left(p+\frac{1}{2}\right) \delta+\frac{\Delta}{2}-R\right]\right]^{2}}{\left[p(p+1)\left(\delta^{2}-\Delta^{2}\right)-W^{2}\right]^{2}+\left[L_{+}^{2}\left(\left(p+\frac{1}{2}\right) \delta+\frac{\Delta}{2}+R\right)+L_{-}^{2}\left(\left(p+\frac{1}{2}\right) \delta+\frac{\Delta}{2}-R\right)\right]^{2}}
$$

with

$$
\begin{aligned}
& L_{+}=L_{1} \cos \theta+L_{2} \sin \theta \\
& L_{-}=L_{1} \sin \theta-L_{2} \cos \theta \\
& K_{+}=K_{1} \cos \theta+K_{2} \sin \theta \\
& K_{-}=K_{1} \sin \theta-K_{2} \cos \theta .
\end{aligned}
$$

Two resonances appear for

$$
\delta= \pm \sqrt{\Delta^{2}+\frac{W^{2}}{p(p+1)}} .
$$

Since $L_{1} L_{2} \ll|\Delta|, W$ we can consider that $\Delta$ does not have a large variation along each resonance and near the resonances $\mathcal{N}$ can be described by

$$
2 P \frac{\left(\mathcal{K}_{ \pm} \mathcal{L}_{ \pm}\right)^{2}}{\left(\delta \mp \sqrt{\left.\Delta^{2}+\frac{W^{2}}{p(p+1)}\right)^{2}+\mathfrak{L}_{ \pm}^{4}}\right.}
$$


where

$$
\begin{aligned}
& \mathcal{K}_{ \pm}=\frac{K_{1}}{\sqrt{p}}\left\{\frac{1}{2} \pm \frac{\Delta}{2 \sqrt{\Delta^{2}+\frac{W^{2}}{p(p+1)}}}\right\}^{1 / 2} \pm \frac{K_{2}}{\sqrt{p+1}}\left\{\frac{1}{2} \mp \frac{\Delta}{2 \sqrt{\Delta^{2}+\frac{W^{2}}{p(p+1)}}}\right\}^{1 / 2} \\
& \mathfrak{L}_{ \pm}=\frac{L_{1}}{\sqrt{p}}\left\{\frac{1}{2} \pm \frac{\Delta}{2 \sqrt{\Delta^{2}+\frac{W^{2}}{p(p+1)}}}\right\}^{1 / 2} \pm \frac{L_{2}}{\sqrt{p+1}}\left\{\frac{1}{2} \mp \frac{\Delta}{2 \sqrt{\Delta^{2}+\frac{W^{2}}{p(p+1)}}}\right\}^{1 / 2} .
\end{aligned}
$$

The resonant curves have respectively widths $\mathcal{L}_{+}^{2}$ and $\mathcal{L}_{-}^{2}$. When $|\Delta| \gg W / \sqrt{p(p+1)}$ we find again the two isolated resonances with widths $\frac{L_{1}^{2}}{p}$ and $\frac{L_{2}^{2}}{p+1}$. When $W \gg|\Delta|$

$$
\begin{aligned}
& \mathfrak{L}_{+}=\frac{1}{\sqrt{2}}\left(\frac{L_{1}}{\sqrt{p}}+\frac{L_{2}}{\sqrt{p+1}}\right) \\
& \mathfrak{L}_{-}=\frac{1}{\sqrt{2}}\left(\frac{L_{1}}{\sqrt{p}}-\frac{L_{2}}{\sqrt{p+1}}\right) .
\end{aligned}
$$

This description of doubly resonant photoionization shows that, when the interaction between the quasiresonant levels is large, the resonance curves are very different from those which could be predicted if the two resonances were considered as isolated.

In any case the distance,

$$
2 \sqrt{\Delta^{2}+\frac{W^{2}}{p(p+1)}}
$$

between the two resonances is larger than $2|\Delta|$ which would be obtained if the resonances were considered as isolated, and the relative values of the widths of the resonant curves $\mathcal{L}_{+}^{2}$ and $\mathcal{L}_{-}^{2}$ can be very different from the relative values of $\frac{L_{1}^{2}}{p}$ and $\frac{L_{2}^{2}}{p+1}$. The most remarkable cases are $L_{1} \ll L_{2}$ where $\mathcal{L}_{+}^{2}$ and $\mathcal{L}_{-}^{2}$ have the same order of magnitude and $L_{1} \sim L_{2}$ where $\mathcal{L}_{-}^{2}$ and $\mathcal{L}_{+}^{2}$ can have very different order of magnitude. In the first case $\left(L_{1} \ll L_{2}\right.$ or $\left.L_{2} \ll L_{1}\right)$, if we should consider the resonances as well isolated, we should obtain two resonance curves with widths of very different order of magnitude, while a calculation taking into account the interaction between the two quasi-resonant levels gives - two resonance curyes with width of the same order of magnitude. In the second case $\left(L_{1} \sim L_{2}\right)$, if we consider the resonances as well isolated, we obtain two resonant curves with width of the same order of magnitude, while a calculation taking into account the interaction between the two quasi-resonant levels gives two resonance curves with widths of different orders of magnitude.

These situations can occur in numerous cases, because the dependence of $L_{1}$ and $L_{2}$ on the field intensity is different : $L_{1}^{2}$ varies as $\bar{N}^{n-p}$ and $L_{2}^{2}$ varies as $N^{n-p-1}$. It certainly may be possible in most cases to realize the successive situations $L_{1} \ll L_{2}$, $L_{1} \sim L_{2}, L_{2} \ll L_{1}$ by increasing the field intensity. A schematic representation of these situation is illustrated in figure 2 .

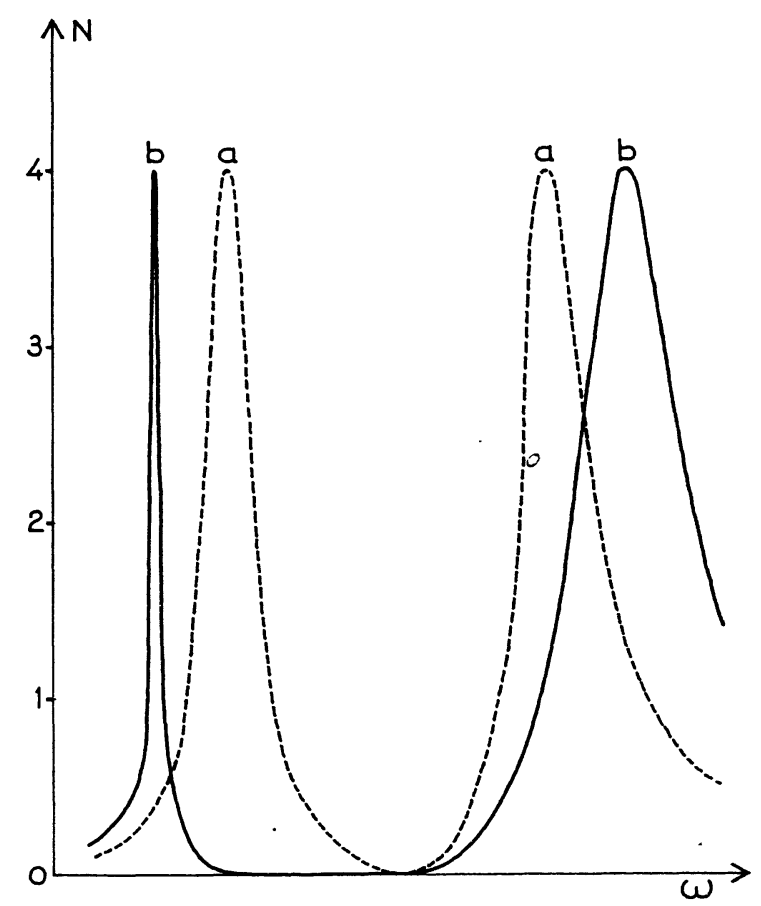

Fig. $2 \alpha$. - Resonance curves calculated fo $L_{1}=L_{2}=\Delta / 10$. a) $W=\Delta / 100 ; b) W=\Delta / 2$.

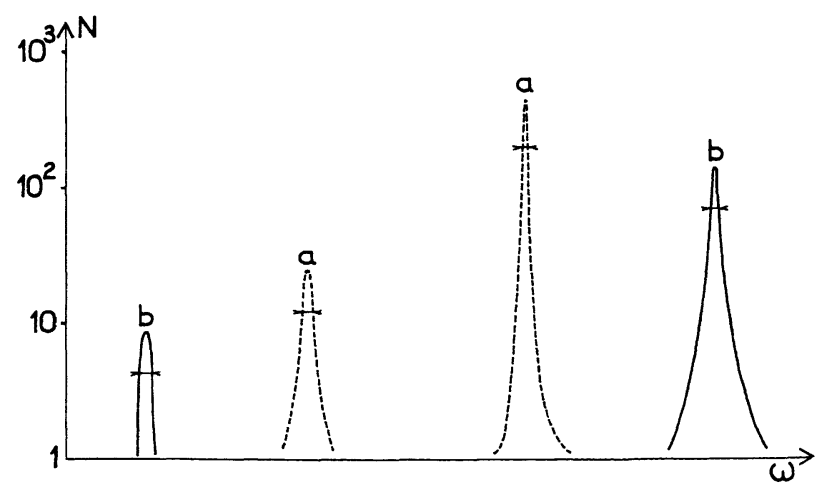

Fig. $2 \beta$ : - Resonance curves calculated for $L_{1}=L_{2} / 2=\Delta / 10$. a) $W=\Delta ; b) W=20 \Delta$. 
The increase of separation between resonances can be easily interpreted by looking at the energy diagram of the dressed atom (see Fig. 3).

The energies of levels

$$
|\mathrm{i} N\rangle\left|\varphi_{1} N-p\right\rangle\left|\varphi_{2} N-p-1\right\rangle
$$

are respectively $\Omega_{\mathrm{i}}+N \omega, \quad \Omega_{1}+(N-p) \omega$, $\Omega_{2}+(N-p-1) \omega$. The variation of the energies of these levels are represented by the straight lines $\mathrm{a}, \mathrm{b}, \mathrm{c}$. If the interaction between $\varphi_{1}$ and $\varphi_{2}$ is neglected, the position of the resonances are given by the intersections $\mathrm{A}$ and $\mathrm{B}$ of $\mathrm{c}$ with respectively $\mathrm{a}$ and $\mathrm{b}$. Because of the strong, interaction between $\varphi_{1}$ and $\varphi_{2}$ the straight lines $\mathrm{a}$ and $\mathrm{b}$ must be replaced by an hyperbola having these lines as asymptotes. The intersections of this hyperbola with the line $\mathrm{c}$ are $\mathrm{C}$ and $\mathrm{D}$. CD is larger than $\mathrm{AB}$ corresponding to the increase of separation between resonances when the interaction $W$ is large.

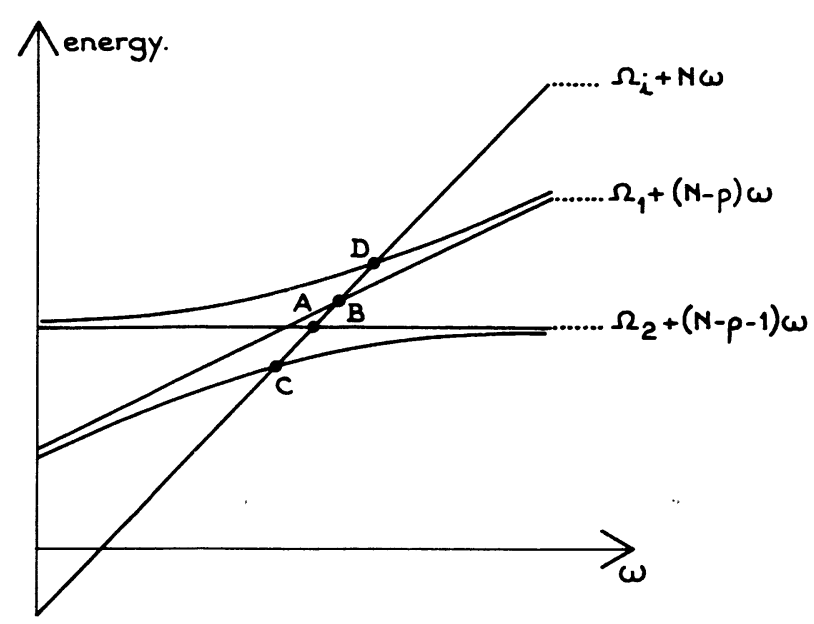

FIG. 3. - Dressed atom energy diagram.

\section{Referęnces}

[1] BAKos, J. S., Adv. electron. electron phys. 36 (1974) 57.

[2] Lambropoulos, P., Adv. at. mol. phys. 12 (1976) 87.

[3] Armstrong, L., Jr., Beers, B. L., Feneuille, S., Phys. Rev. A 12 (1975) $1903-12$.

[4] Faisal, F. H. M., J. Phys. B 9 (1976) 3009-16.
[5] Dixit, S. N., Lambropoulos, P., Abstr. Int. Conf. Multiphoton Processes, Rochester (1977).

[6] Crance, M., to be published in J. Phys. B.

[7] Morellec, J., Normand, D., Petite, G., Phys. Rev. A 14 (1976) 300-12. 